\title{
SwissDRG: 3 Jahre alt und schon Routine? Eine Antwort
}

\section{Christian Hess}

Dr. med., Chefarzt von 1988-2012, Akademie Menschenmedizin

Im Anschluss finden Sie eine Stellungnahme von P.-F. Cuénoud.

1 Cuénoud PF. SwissDRG 3 Jahre alt und schon Routine? Schweiz Ärztezeitung. 2015;96(5):127.

Korrespondenz: Dr. med. Christian Hess Husertalstrasse 9 CH-8925 Ebertswil ch.hess1[at]gmail.com www.menschenmedizin.ch
Herr Cuénoud, so macht es den Eindruck, ringt mit Worten und Gedanken, um die doch sehr durchzogenen Erfahrungen mit den Fallkostenpauschalen als erfolgreich bilanzieren zu können. Das ist ja verständlich, wenn man im Kopf hat, wie er sich für dieses System eingesetzt hat und nach wie vor engagiert. Doch schauen wir genauer hin. Was waren die Versprechen damals und was sind die Resultate heute? 1. Mehr Transparenz war das Hauptversprechen. Hier muss selbst Herr Cuénoud eingestehen, dass es damit nicht zum Besten steht. Jeder Kanton hat eine andere Basalrate und sogar innerhalb der Kantone gibt es nicht selten auch noch Unterschiede (was notabene dazu führt, dass Patient(inn)en im gleichen Kanton für die gleichen Interventionen in verschiedenen Spitälern unterschiedlich viel Selbstbehalt zahlen müssen). Jeder Kanton bezahlt zudem gemeinwirtschaftliche Leistungen nach eigenem Gutdünken, und jeder Kanton entscheidet, ob er bei «seinen» Spitälern Investitionen weiterhin mitfinanziert oder nicht. Viel verwirrender kann ein System kaum sein. 2. Weniger Kosten zu verursachen war ursprünglich ebenfalls ein Hauptargument. Auch da gibt es wenig Positives zu vermelden. Die Kosten steigen unvermindert, was auch nicht anders zu erwarten war - man hätte es im Voraus von Deutschland lernen können. Insbesondere in der Chirurgie nehmen die Operationen und damit die "Fallzahlen" der Patienten unter diesem System massiv zu. Dies kann nur durch weichere Indikationsstellung erklärt werden (vgl. dazu Comparis, 6.8.2014: Geld statt Gesundheit: Ärzte gestehen unnötige Operationen). Begünstigt wird dieser Trend noch durch die häufigen Bonusverträge, die in der Chirurgie inzwischen alltäglich sind.

3. Mehr Qualität war das dritte Stichwort. Es ist zugegebenermassen schwierig zu zeigen, ob diese zu- oder abnimmt. Was wir aber wissen, ist, dass die Rehabilitationskliniken unter Druck gekommen sind, weil sie viel mehr Schwerkranke zugewiesen bekommen, (die noch gar nicht rehabilitierbar sind und) für die sie grundsätzlich nicht der richtige Ort sind. Dazu kommt, dass durch die Mengenausweitung im operativen Bereich reine Outcome-Messungen natürlich gut abschneiden (man hat ja Gesündere operiert), was zu einer Beschönigung der Bilanz führt. Die so dringend benötigte Indikationsqualität, von uns («DRGMoratorium») als erste gefordert, ist mittlerweile zwar viel diskutiert, aber nirgends wirklich etabliert.

4. Mehr Wettbewerb. Dieses Versprechen ist in begrenztem Masse erreicht. Aber ist das ein Gewinn? Die Beantwortung dieser Frage scheint mittlerweile zu einer rein ideologischen Diskussion verkommen zu sein. Tatsache ist, dass aufgrund des Wettbewerbs infrastrukturell in Millionenhöhen aufgerüstet wird. Der Versorgungsauftrag wird dabei zunehmend aus den Augen verloren. Man konzentriert sich auf "rentable» Bereiche und forciert die Mengenausweitung, um vorgegebene Budgetziele zu erreichen.

Neben diesen Hauptargumenten der damaligen DRGBefürworter kommen nun, wie von Cuénoud beschrieben, weitere zweifelhafte kollaterale Folgen zum Tragen. Da heisst es doch ohne Umschweife, dass innerhalb eines Jahrzehnts ein ganzes Absolventenjahr von Ärztinnen und Ärzten in die Administration abwandert. Es passiert also etwas, das wir uns beim drohenden und z.T. schon bestehenden Ärztemangel am allerwenigsten leisten können.

Die Bürokratisierung, sie wird in Cuénouds Artikel ebenfalls thematisiert, allerdings mit dem hoffnungsvollen Satz ergänzt, dass Spitalärzte deshalb keinesfalls ihre klinischen Aufgaben vernachlässigen sofern sie dazu noch Zeit haben, ist man geneigt zu denken. Von der Pflege und den vielen anderen Berufsgruppen ist schon gar nicht mehr die Rede. Das schweizerische Gesundheitswesen kostet jährlich rund 2,3 Milliarden CHF mehr und das bei einschneidenden Sparmassnahmen am Patientenbett! Und wo fliessen diese Gelder hin? Natürlich in die Administration.

Was Einführung, Weiterentwicklung, Kontrolle und interne Optimierungen dieses Systems die Allgemeinheit kosteten und weiterhin kosten, wird nicht kommuniziert. Es sind aber seit Beginn dieser Umgestaltung Hunderte von Millionen Franken. Diese fehlen an anderer Stelle. Die Frage liegt auf der Hand, ob man für so viel Geld nicht patientenwirksamere Reformen 
hätte realisieren können. Die ethische Umkehr, die dieses System erwirkt, macht Sorgen: Wurden früher Ressourcen für leidende, kranke Menschen von der Gesellschaft zur Verfügung gestellt, so werden neu
Patienten mit ihren Leiden und Krankheiten zur Gewinnoptimierung missbraucht.

Wenn man all das als Erfolg verkaufen will, dann liest es sich in etwa so wie im Artikel von Cuénoud. 